\title{
A Case Report of Spinal Cord Injury Patient From a High Velocity Gunshot Wound to the Lumbar Spine
}

\author{
Juyong Kim, MD, Je Ho Kim, MD, Moon Suk Bang, MD
}

Department of Rehabilitation Medicine, Seoul National University College of Medicine, Seoul, Korea

We report on operational and rehabilitation management, as well as the outcome, of a patient who with sustained spinal cord injury from a high velocity gunshot wound to the lumbar spine. More specifically, a patient with a gunshot wound to the spine is more likely to sustain a complete injury and have a poor prognosis. As such, there should be concerns regarding associated and extended injuries related to bullet fragmentation as well as the possibility of long-term sequelae.

Keywords Gunshot wound, Spinal cord injury, Forensic ballistics

\section{INTRODUCTION}

Gunshot wound is one of the leading causes of spinal cord injuries in the world, accounting for $13 \%$ to $43 \%$ of such injuries [1]. In addition, spinal cord injuries following gunshot wounds show a higher incidence of complete injury with worse outcomes than that resulting from blunt trauma [2].

Generally, gunshot wounds are divided into two categories-those resulting from high velocity weapons typically used for military purposes; and wounds from lowvelocity weapons such as pistols and handguns. Between

Received November 10, 2010; Accepted April 25, 2012

Corresponding author: Moon Suk Bang

Department of Rehabilitation Medicine, Seoul National University College of Medicine, 101 Daehak-ro, Jongno-gu, Seoul 110-744, Korea Tel: +82-2-2072-2925, Fax: +82-2-743-7473, E-mail: msbang@snu.ac.kr

(c) This is an open-access article distributed under the terms of the Creative Commons Attribution Non-Commercial License (http://creativecommons. org/licenses/by-nc/3.0) which permits unrestricted noncommercial use, distribution, and reproduction in any medium, provided the original work is properly cited.

Copyright $\odot 2013$ by Korean Academy of Rehabilitation Medicine the two groups, nonmilitary gunshot wounds tend to result from low velocity weapons. However, injuries from high velocity weapons among the civilian population have recently been on the rise. Overall, cases of gunshot wounds to the spine are relatively rare in Korea, except for the veterans of the Korean War and the Vietnam War. In Korea, there was only one case report on a patient with spinal cord injury due to a low velocity gunshot wound to the thoracic spine [3]. Here, we describe the first case of a Korean man with spinal cord injury caused by a high velocity gunshot wound.

\section{CASE REPORT}

A 40-year-old man became paraplegic after a gunshot wound to the lumbar spine. The injury occurred when he came under fire during a shooting rampage while he was abroad. The perpetrator had fired an AR-15 rifle ArmaLite Model 15 (Colt Inc., Hartford, CT, USA), and the bullet went through the victim's back. After the incident, his bilateral lower limbs showed no muscle contraction 
with sensory loss. The patient underwent explorative laparotomy and was diagnosed with injuries to the spinal cord and aorta. He was transferred to our hospital on the second post-traumatic day. A computed tomography (CT) scan revealed a burst fracture of the first lumbar vertebra, resulting from penetration by a bullet from the gunshot through the right paraspinal muscles and L1-2 intervertebral disc (Fig. 1). The bullet was fragmented, and one of

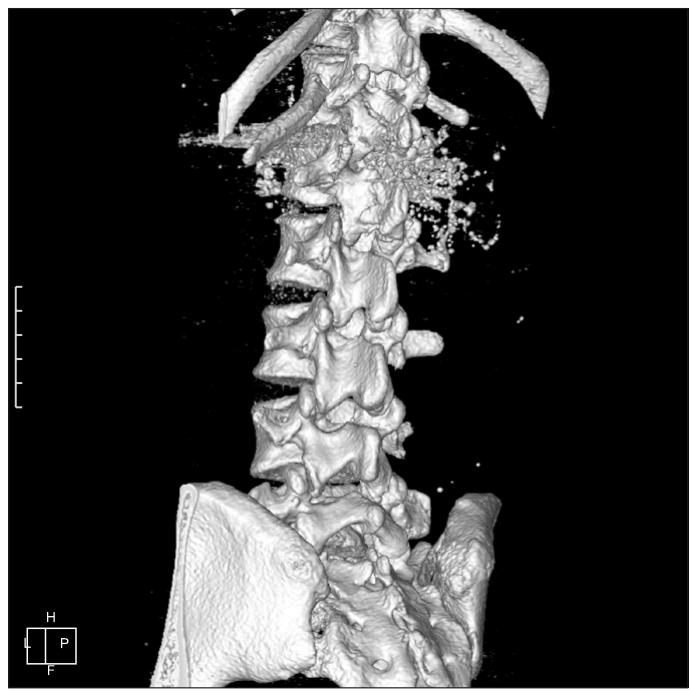

Fig. 1. Preoperative 3-dimentional reconstructed computed tomography shows a burst fracture of the first lumbar vertebra.

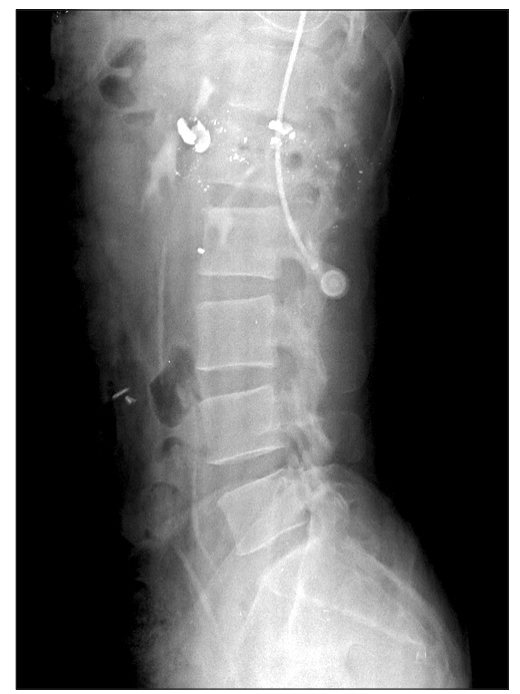

Fig. 2. Preoperative simple $\mathrm{X}$-ray of the spine shows 2 large bullet fragments: 1) T12-L1 disc level, a $0.8-\mathrm{cm}$ sized bullet fragment and 2) prevertebral space of $\mathrm{Ll}$, a $1.0-\mathrm{cm}$ sized bullet fragment. the largest fragments was lodged in the T12-L1 disc. Another large fragment was located between the right vertebral body and aorta at the level of the renal arteries (Fig. 2). Apart from bowel contusion, there was no evidence of intra-abdominal trauma such as active bleeding or bowel perforation on the patient's CT images.

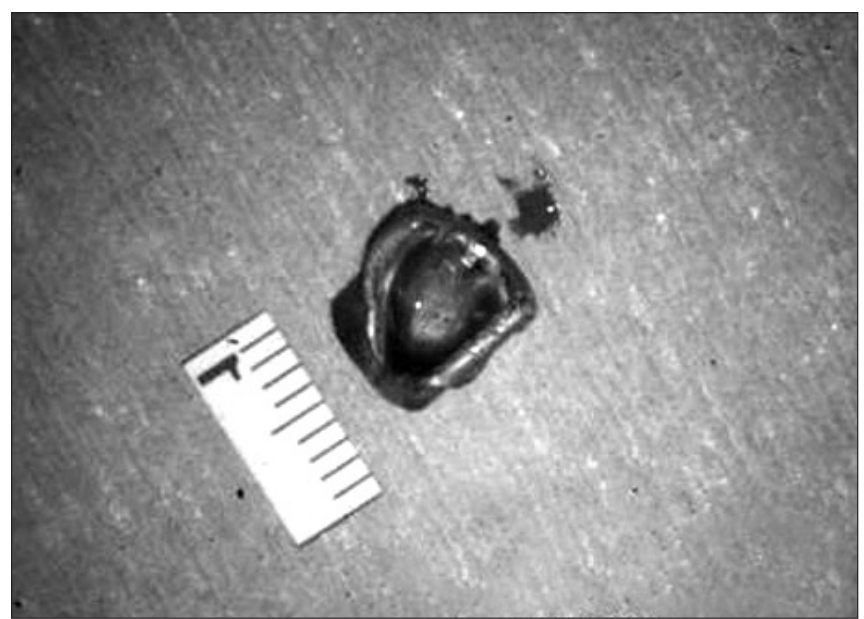

Fig. 3. Photograph of a bullet fragment in the T12-L1 disc space.
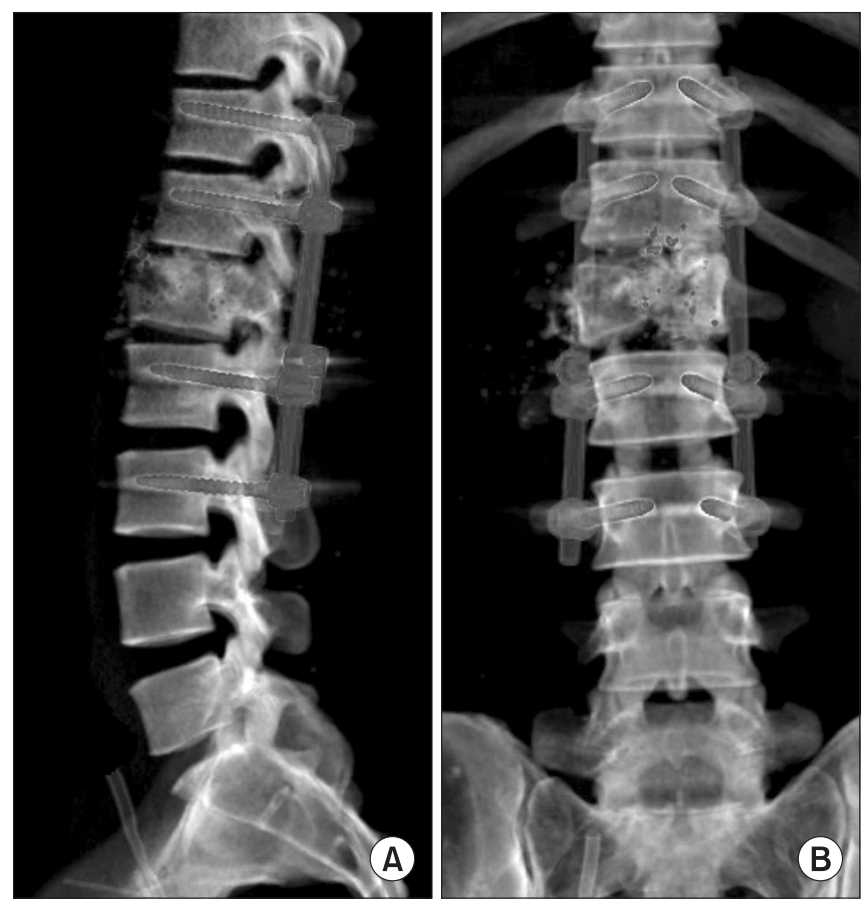

Fig. 4. Postoperative 3-dimentional computed tomography of the spine shows posterior fixation of T11-L3 and remnant bullet fragments in the spinal canal of the L1 level (A, lateral view; B, anteroposterior view). 
The patient underwent $\mathrm{Ll}$ decompressive laminectomy, partial corpectomy, removal of the bullet and T11-L3 posterior fixation. The dura in the level of the first lumbar vertebra was severely damaged, and most parts of the cauda equina were severed from the conus medullaris. A $0.8-\mathrm{cm}$ bullet fragment at the T12-L1 disc level was removed (Fig. 3). Subsequently, a rupture of the abdominal aorta was treated by a vascular surgeon. A $1.0-\mathrm{cm}$ bullet fragment just anterior to the first lumbar vertebral body was also removed. However, massive bleeding occurred following removal, so aorta graft interposition with polytetrafluoroethylene was performed after resection of the aorta between the renal artery and the superior mesenteric artery. A possibility of a second insult to the spinal cord due to infarction caused by massive bleeding was considered, although it could not be confirmed. A postoperative spinal CT scan revealed multiple bone remnants and bullet fragments in the L1-2 intervertebral disc and spinal canal space (Fig. 4).

The patient subsequently began range of motion exercise at bedside on the 13th post-traumatic day and tilting table exercise in the gym on the 18th day.

On the 39th post-traumatic day, the patient was transferred to the Department of Rehabilitation. His neurological level of injury was determined as T12 according to the American Spinal Injury Association Impairment Scale (AIS)-A. Sensory examination revealed sensory loss in $\mathrm{L} 2$ and below. The manual muscle test showed grade 0 in all the muscles of the bilateral lower limbs. Deep tendon reflexes of the bilateral knees and ankle joints were decreased and muscle tones of bilateral lower extremities were flaccid. The bulbocavernosus reflex was absent. His bladder and bowel sense decreased, and urodynamic examination revealed detrusor areflexia with coordinated sphincter.

On the 53th post-traumatic day, the patient underwent an electromyographic study. No responses were observed in motor nerve conduction study, $\mathrm{H}$ reflex, $\mathrm{F}$ wave, somatosensory evoked potential and bulbocavernosus reflex. The sensory nerve conduction study showed a normal value. Needle electromyography of all the muscles of the bilateral lower extremities showed severe to profound abnormal spontaneous activities with no motor unit action potential. The results were compatible with polyradiculopathy or the anterior horn cell lesion below L1, and there was no evidence of reinnervation.
The patient complained of severe neuropathic pain in the bilateral lower extremities (visual analogue scale [VAS], 7 to 8 ). Neuropathic pain was characterized by tingling and squeezing, which were aggravated at night. Although transcutaneous electrical nerve stimulation (TENS) and many kinds of analgesics including gabapentin (900 mg/day), pregabalin (450 mg/day), amitriptyline (25 mg/day), clonazepam ( $1 \mathrm{mg} /$ day), oxycodone (20 mg/ day), and fentanyl patch $(50 \mu \mathrm{g} / \mathrm{hr})$ were tried simultaneously and alternatively, he continued to suffer from pain that waxed and waned in the range of 4 to 8 on the VAS.

On the 55th post-traumatic day, he performed parallelbar (p-bar) gait with both knee-ankle-foot orthosis (KAFO), in parallel with the Walking Index for Spinal Cord Injury (WISCI) level 1 . On the 90th post-traumatic day, he managed axillary crutch gait with both KAFO in the p-bar. Seven months after the trauma, however, there was no recovery in the neurological level of his injury according to neurologic examinations including the manual muscle test and sensory exam compared with the initial evaluation. Moreover, the patient's endurance and stability of gait function remained poor to the point that he could only manage the p-bar gait for 2 cycles and crutch gait for one quarter of a cycle in the p-bar (WISCI level 3). He showed no significant improvement in the activities of daily living, measured by the Korean Spinal Cord Independence Measure (KSCIM) score over the course from the 39th to the 81st post-traumatic day (from 32 to 35 points). Improvement was shown in bathing, dressing and motion in bed.

\section{DISCUSSION}

In the present report, we described a patient with injuries to the spinal cord and aortic wall from a high velocity gunshot wound. Such injuries usually occur in soldiers and involve wounds from gunshots fired at speeds greater than 2,000-3,000 feet per second, whereas low velocity injuries result from gunshots at 1,000-2,000 feet per second. The characteristics of high velocity gunshot injury are comminuted fractures, devitalized soft tissue, and periosteal stripping. The extent of bullet fragmentation also increases concomitantly with velocity, resulting in more severe destruction due to the wider range of damage to tissue compared to low velocity gunshot wounds [4]. Consequently, high velocity gunshots correlate with 
higher mortality and morbidity than low velocity gunshots [5].

The AR-15 rifle is a high velocity weapon; its bullet can reach speeds of up to 3,200 feet per second. Accordingly, our patient suffered from a burst fracture of the first lumbar vertebra due to the high power exuded by the weapon, which resulted in complete paraplegia and severe neuropathic pain as well as an aortic injury resulting from an impacted bullet fragment in the posterior wall of the abdominal aorta. Furthermore, multiple remnants of bullet fragments lodged in the T12-L1 disc and L1 prevertebral areas could not be removed.

There have been articles describing whether surgery can produce neurologic benefits. For example, Stauffer et al. [6] demonstrated that no neurological difference was observed in either surgery or nonoperative management for complete or incomplete spinal cord injuries. Both groups had similar rates of causalgia [6]. To deal with lesions associated with intracanal bullets, Waters and Adkins [7] reported a statistically significant improvement in motor function after surgical removal and decompression from the T12 to L4 levels, in comparison with nonoperative management. Although fractures resulting from gunshot wounds are usually not unstable and rarely require stabilization [1], our patient had a burst fracture of the first lumbar vertebra due to high energy from the bullet, requiring operation. Furthermore, some case reports have supported that bullet migration can cause neural deficits lasting from several months to years after a gunshot injury [1]. In the present case, multiple remnants of bullet fragments in the spinal canal may potentially lead to a continuous, diffuse inflammatory reaction, hindering neurologic recovery [1]. Therefore, even with proper operative management, there still exists a possibility of long term negative effects associated with residual fragments of the bullet.

In the past, the gunshot etiology of spinal cord injuries was unrelated to the measurements of pain and the Functional Independence Measure (FIM) scores [8,9]. However, complete lesions are more common in spinal cord injuries caused by gunshots than other etiologies [2]. The patient had a complete injury in the levels of the conus medullaris and cauda equina along with sustained extensive wounds, all of which caused him to suffer severe neuropathic pain. The neurologic outcome of this case, which involved a complete lesion, was poor, as is typical for a patient suffering from a complete spinal cord injury.

Magnetic resonance imaging (MRI) is generally regarded as the best method for assessing ordinary spinal cord injuries. However, the strong magnet used in the procedure can cause bullet migration and lead to additional neurologic damage. Finitsis et al. [10] have retrospectively reported that the use of MRI on patients with retained metallic ballistic fragments in the spinal region can yield more information than other imaging techniques. The study further asserted that no untoward effects were seen [10]. Nevertheless, the safety of MRI technology has yet to be proven by any prospective controlled scientific study [1]. In the present case, we decided not to perform MRI on the patient since a bullet fragment lodged in his spinal canal could easily migrate to other regions.

In conclusion, spinal cord injury patients caused by high velocity gunshot wounds have relatively poor prognosis due to worse neurological improvement. Therefore, careful evaluations of gunshot wound patients are required by physiatrists, especially in terms of gunshot velocity, lesion of injury and presence of remnant bullets or other accompanying injuries for proper goal setting for rehabilitation and management.

\section{CONFLICT OF INTEREST}

No potential conflict of interest relevant to this article was reported.

\section{REFERENCES}

1. Bono CM, Heary RF. Gunshot wounds to the spine. Spine J 2004;4:230-40.

2. Kaufman HH, Pait TG. Gunshot wounds to the spine. Contemp Neurosurg 1993;15:1-6.

3. Lim SJ, Kim YT, Ha HG. An operative case of air-gunshot wound to the thoracic spine. J Korean Neurosurg Soc 1997;26:584-8.

4. Bartlett CS. Clinical update: gunshot wound ballistics. Clin Orthop Relat Res 2003;(408):28-57.

5. Ordog GJ, Wasserberger J, Prakash A, Balasubramaniam S. Civilian gunshot wounds: determinants of injury. J Trauma 1987;27:943-7.

6. Stauffer ES, Wood RW, Kelly EG. Gunshot wounds of the spine: the effects of laminectomy. J Bone Joint 
Surg Am 1979;61:389-92.

7. Waters RL, Adkins RH. The effects of removal of bullet fragments retained in the spinal canal: a collaborative study by the National Spinal Cord Injury Model Systems. Spine (Phila Pa 1976) 1991;16:934-9.

8. Putzke JD, Richards JS, Devivo MJ. Gunshot versus nongunshot spinal cord injury: acute care and rehabilitation outcomes. Am J Phys Med Rehabil 2001;80:366-70.
9. Waters RL, Adkins RH, Sie I, Cressy J. Postrehabilitation outcomes after spinal cord injury caused by firearms and motor vehicle crash among ethnically diverse groups. Arch Phys Med Rehabil 1998;79:123743.

10. Finitsis SN, Falcone S, Green BA. MR of the spine in the presence of metallic bullet fragments: is the benefit worth the risk? AJNR Am J Neuroradiol 1999;20:3546. 